\title{
THE MEASURING SYSTEM FOR EVALUATION OF PARAMETERS OF INTEGRATED MACH-ZEHNDER MODULATOR
}

The paper deals with some issues linked to practical application of the analog intensity electro-optic modulator of Mach-Zehnder type. An accurate adjustment and long-term stabilization of optimal operating point of the modulator belongs to the most severe problems linked to implementation of the appliance like this. The paper describes functionality of Mach-Zehnder modulator, highlights some of the problems the designer is faced to and presents the methodology that has been used to measure parameters of the modulator that are not specified in the manufacturer data-sheet and might be helpful to solve the problem.

\section{Introduction}

A specialized electro-optic remote-antenna system is under development at our department. The system should be able to transmit very weak and broadband analog signals via optical fibre. In terms of the most critical parameters there is a necessity: a) to cover the frequency range from virtual $\mathrm{DC}$ up to $2.5 \mathrm{GHz}$ at least, b) to ensure $50 \Omega$ matched input and output, c) to ensure high level of galvanic insulation between input and output, d) to achieve positive gain of the system and e) to maintain a high fidelity of transmitted signal. Probably the only way to achieve the features mentioned above simultaneously is to implement the system by using an external modulation technique. A simplified structure of the proposed system can be seen in Fig. 1. The broadband external modulating unit (Intensity modulator) forms its core and vital part. The modulating unit shall be based on the analog intensity electrooptic modulator of Mach-Zehnder type (MZM).

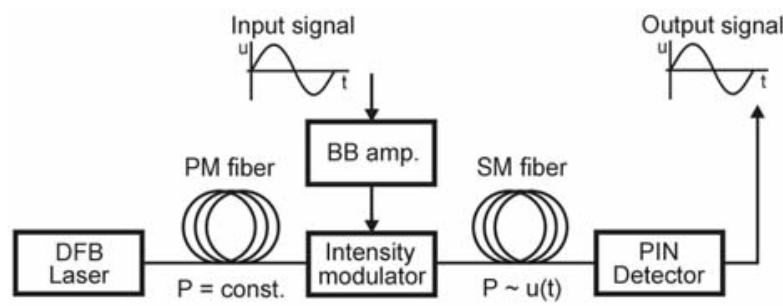

Fig. 1 Electro-optic remote antenna system

During the last few decades a lot of external electro-optic modulators have been developed and upgraded. Nowadays, some of them are even commercially available in integrated form. A modulator of Mach-Zehnder type belongs to the most mature ones.
Basically, it can be described as two-arm interferometer integrated in convenient material. $\mathrm{A} \mathrm{LiNbO}_{3}$ substance seems to be the most widespread substrate in use, due to its good electro-optic features. The waveguide arms are made of indiffused titanium for example. Both the arms are connected by means of two Y-junctions, see Fig. 2. The junction $Y 1$ is the input one and ensures balanced split of incoming light into the arms. The junction Y2 is the output one and thus recombines the outgoing light-waves.

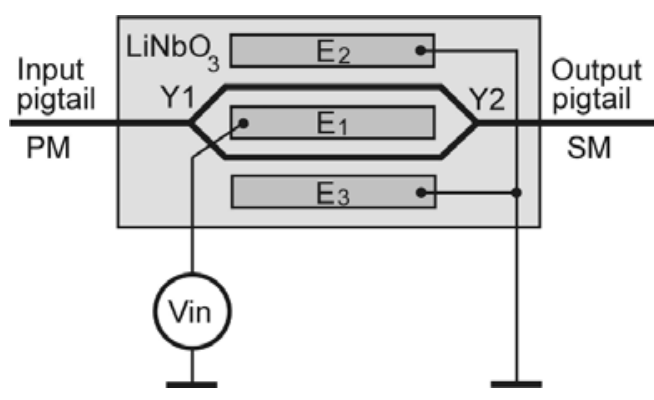

Fig. 2 Basic configuration of $M Z M$

The integrated structure is equipped with system of electrodes $\left(E_{1}, E_{2}, E_{3}\right)$ appropriately positioned above the waveguides. A voltage $V_{\text {in }}$ applied across the electrodes induces electrical field around them and thus in the electro-optic material too. The field changes refractive index of the active material. The phenomenon produces the phase shift of the light waves travelling through the particular arms. Due to the recombination of the light-waves in the $Y_{2}$ junction, the interference of the light arises. If the phase difference between the waves is zero, the recombination is constructive and maximum amount of the light is leaving the output pigtail. If the phase shift difference is exactly $180^{\circ}$, the recombination is destruc-

\footnotetext{
* Jiri Svarny

Department of Technologies and Measurements, Faculty of Electrical Engineering, University of West Bohemia, Univerzitni 8, Plzen,

Czech Republic, E-mail: svarny@ket.zcu.cz
} 
tive and thus the outgoing light is attenuated maximally. By means of gradual transition between these two extremes, the smooth change of light intensity can be achieved. That is the way, how to modulate intensity of optical power by means of applied voltage.

\section{Theoretical Background}

Unfortunately, the MZM transfer function describing dependency of outgoing optical power $P_{o}$ on driving voltage $V_{i n}$ is not linear.

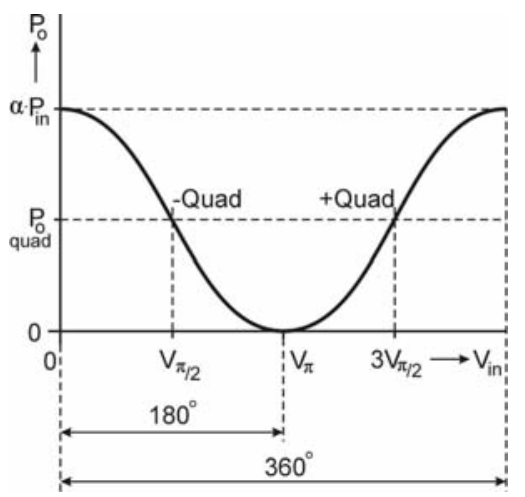

Fig. 3 Typical transfer function of an ideal MZM

In accordance with Fig. 3, the transfer function of the MZM can be described by means of cosine. The function (1) is periodical and the basic pattern (Fig. 3 ) repeats as the voltage $V_{\text {in }}$ grows up,

$$
P_{o}=\frac{P_{i n} \alpha}{2}\left[1+\cos \left(\frac{\pi V_{i n}}{V_{\pi}}\right)\right]
$$

where $P_{o}$ and $P_{i n}$ are output and input optical power respectively, $V_{\text {in }}$ is input voltage and $V_{\pi}$ is so called half-wave voltage representing a value of $V_{\text {in }}$ needed to change output power of MZM from one extreme to opposite one. The $\alpha$-coefficient represents insertion loss of the modulator.

To operate the MZM properly, it is necessary to set-up the operating point by appropriate value of DC component of $V_{\text {in }}$ (bias voltage $V_{\text {bias }}$ ). In case of analog optical link the desired value corresponds to the point, where the electro-optic gain is ultimate. The value of desired bias voltage can be found by substituting $V_{\text {bias }}$ for $V_{\text {in }}$ in (1), solving the second derivative of the function and putting it equal zero (2).

$$
\frac{d^{2} P_{o}}{d V_{\text {bias }}^{2}}=-\frac{P_{\text {in }} \alpha \pi^{2}}{2 V_{\pi}^{2}} \cos \left(\frac{\pi V_{\text {bias }}}{V_{\pi}}\right)=0
$$

From the equation (2) a condition (3) can be derived, provided $k$ is an integer.

$$
V_{\text {bias }}=\frac{V_{\pi}}{2}(2 k+1)
$$

As far as the input voltage $V_{\text {in }}$ is obtained as a superposition of DC bias voltage $V_{\text {bias }}$ and modulating AC voltage $V_{\text {mod }}(4)$ the function (1) can be decomposed to formula (5) [1].

$$
\begin{aligned}
& V_{\text {in }}=V_{\text {bias }}+V_{\text {mod }} \\
& P_{o}=\frac{P_{\text {in }} \alpha}{2}\left[1+\cos \left(\frac{\pi V_{\text {bias }}}{V_{\pi}}\right) \cos \left(\frac{\pi V_{\text {mod }}}{V_{\pi}}\right)\right]- \\
& -\frac{P_{\text {in }} \alpha}{2}\left[\sin \left(\frac{\pi V_{\text {bias }}}{V_{\pi}}\right) \sin \left(\frac{\pi V_{\text {mod }}}{V_{\pi}}\right)\right]
\end{aligned}
$$

Provided the DC bias voltage $V_{\text {bias }}$ is kept at optimal value (3), the formula (5) can be simplified to the form (6). The particular, desired operating point is called the quadrature point of the modulator.

$$
P_{o}=\frac{P_{i n} \alpha}{2}\left[1-\sin \left(\frac{\pi V_{\text {mod }}}{V_{\pi}}\right)\right]
$$

A setting of the operating point to the quadrature one brings next important benefit. Under the condition a supreme linearity of transfer function is achieved. If the modulating voltage is a low amplitude signal $\left(V_{\text {mod }}<<V_{\pi}\right.$ ), the function (6) can be rewritten in the linearized form (7).

$$
P_{o} \cong \frac{P_{i n} \alpha}{2}\left(1-\frac{\pi V_{\text {mod }}}{V_{\pi}}\right)
$$

In fact, there are some important differences between a simplified modulator depicted in Fig. 2 and a real, commercially available, integrated intensity MZM. The driving electrodes are usually separated into two systems in order to facilitate the implementation of the MZM. Typically one system serves as a DC bias port to adjust the operating point. The second system is connected to $\mathrm{RF}$ input and it is adapted to process $\mathrm{AC}$ high frequency signals up to several $\mathrm{GHz}$ or even tens of $\mathrm{GHz}$ (depending on particular model).

\section{Problem Definition}

In real situation the chart depicted in idealized figure Fig. 3 is shifted to the left or right alongside the $V_{\text {in }}$ axes. The disproportion originates in difference between lengths of the waveguide arms and is caused by technological limits. In real application it is necessary to compensate the intrinsic offset $V_{\text {off }}$ by additional adjustment of DC bias voltage. The value of particular offset is not specified in MZM data-sheet and has to be found by measurements

Furthermore, even more severe problem originates in the fact that biased operating point of MZM suffers from infinite time-drift. The reasons for this behaviour are inherent in complex combination of pyroelectric, photorefractive and photoconductive phenomenon taking action in electro-optic materials of MZM devices [2].

Despite of rapid progress in development of MZMs, the impact of the phenomenon mentioned above has not been resolved in a satisfactory manner yet. That is why, the operating point has to 


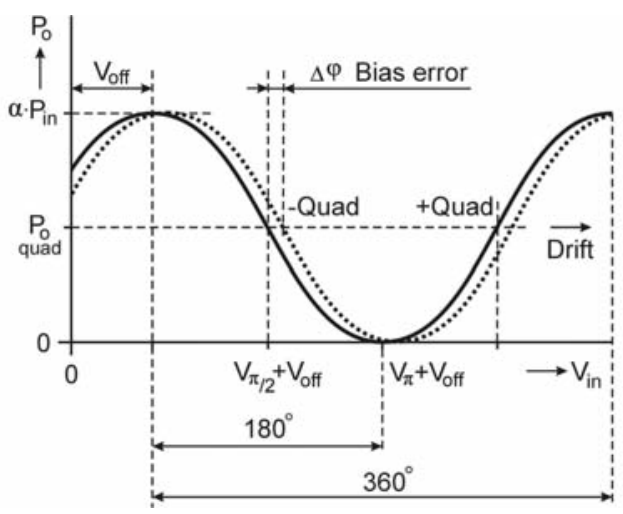

Fig. 4 The MZM transfer chart affected by intrinsic offset and time-drift

be monitored and adjusted from time to time. In case of long-term operating MZMs it is felicitous to implement some automatic feedback to adjust the operating point continuously. There have been developed various methods to keep the operating point at desired position. One of them is depicted in Fig. 5. The method works on principle of automatic adjustment of DC bias voltage in order to keep the value of average output optical power at constant level. For this purpose the optical power exiting the modulator must be monitored. Traditionally an optical tap coupler with external photodiode (PD) is used to sample small percentage of output optical power. Thanks to the linearity of the monitor PD transfer characteristic the current generated by the PD is proportional to the output power. The current is used as an input signal for the bias controller (Feedback circuit). The bias controller works as an efficient integrator. The integrated value of the monitor PD signal is compared to the desired predefined value and difference is used to adjust the bias voltage in proper manner.

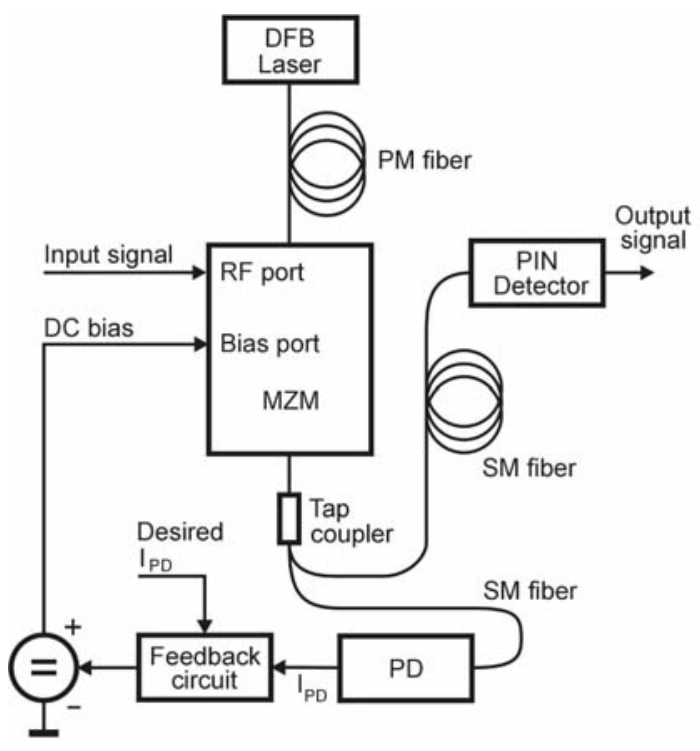

Fig. 5 Constant output power stabilizing method implementation [3]
Nowadays, some of the manufacturers provide modulators with integrated photodiode. The inner PD enables to monitor output optical power and the external tap coupler is no longer needed then. In that case the transfer chart of the inner PD as well as the PD responsivity represents very valuable information for development of prospective feedback system.

\section{Experimental Part}

The integrated intensity MZM, FA20 type [4] was chosen here as a key component of the system. It is a typical $\mathrm{LiNbO}_{3}$ interferometric modulator with titanium indiffused waveguides. The device is tailored to work in infrared range from 1525 up to $1615 \mathrm{~nm}$. The modulator is remarkable thanks to its low $V_{\pi}$ voltage, low insertion loss and wide bandwidth ( $S_{21} 13 \mathrm{GHz}$ for $-3 \mathrm{~dB}$ drop). The model uses separated RF and DC bias ports and it is equipped with monitor photodiode integrated in radiating mode. That means the photodiode current decreases as the output optical power increases. The input pigtail is single mode, polarization maintenance (SM/PM) Panda fiber ended with standard, polished connector (SC/PC). The output pigtail is single mode (SM) fiber ended with $\mathrm{SC} / \mathrm{PC}$ connector too.

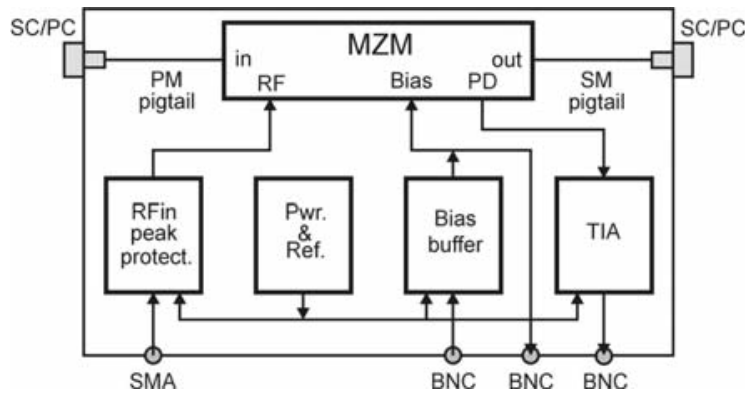

Fig. 6 The block diagram of the base-board of the MZM

There has been designed and developed testing base-board for the external MZM. The block diagram of the device can be seen in Fig. 6 and its final implementation is in Fig. 11. Its primary purpose was to provide safe measurements of parameters of the particular modulator because some of the parameters are not included in the data-sheet. Simultaneously, the board was designed in such a way it could be easily expanded in the future. It is expected that there will be necessity to equip the base-board with additional circuitry (broadband preamplifier of RF port, circuit for stabilization of operating point, etc.).

The base-board includes linear voltage regulator, reference voltage source, DC bias input buffer, the monitor photodiode transimpedance amplifier (TIA) with adjustable gain, protective circuits for RF port and GPO to SMA connector adaptor. By means of the appliance the value of responsivity of monitor photodiode, transfer characteristic of the modulator, $P_{o}=f\left(V_{\text {bias }}\right)$ and monitor photodiode characteristic, $I_{p d}=f\left(P_{o}\right)$ were measured. 
All of the measured charts and parameters were obtained using optical source that is expected to be an integral part of the future electro-optic remote antenna system. The optical source is thermally stabilized DFB laser that was designed and manufactured during the previous stage of the project. It generates output optical power of $21.68 \mathrm{~mW}$ with long-term (3 hour) stability better than $\pm 0.009 \mathrm{~dB}[5]$.

While measuring the characteristics the voltage $V_{\text {in }}$ applied to the DC bias input was considered to be the only independent variable. In order to ensure mutual correlation among measured charts it was necessary to measure all the parameters with sufficient velocity (rejection of the bias drift influence). The ideal method is to measure all the charts in one go.

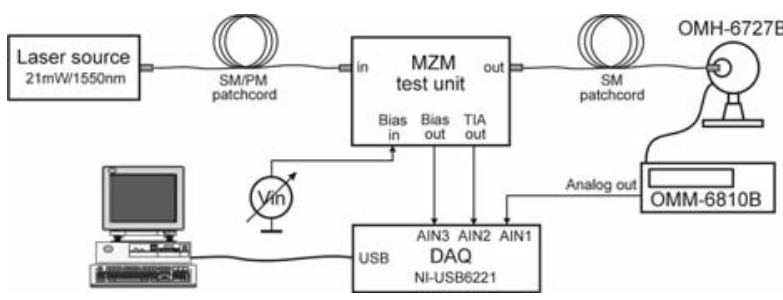

Fig. 7 The measuring system designed for evaluation of parameters of the $M Z M$

That is why the measurements were done by means of system depicted in Fig. 7. The output optical power was measured by means of optical multimeter OMM-6810B equipped with OMH6727B sensing head [6]. The multimeter disposes with analog output generating voltage that is proportional to the value of measured optical power. The output voltage was recorded by means of DAQ system NI-USB-6221 and PC working under LabVIEW application. Simultaneously, a signal from trans-impedance amplifier and DC bias voltage were recorded too. The RF input of MZM was unplugged. The measurements were done at the room temperature of $23 \pm 1{ }^{\circ} \mathrm{C}$.

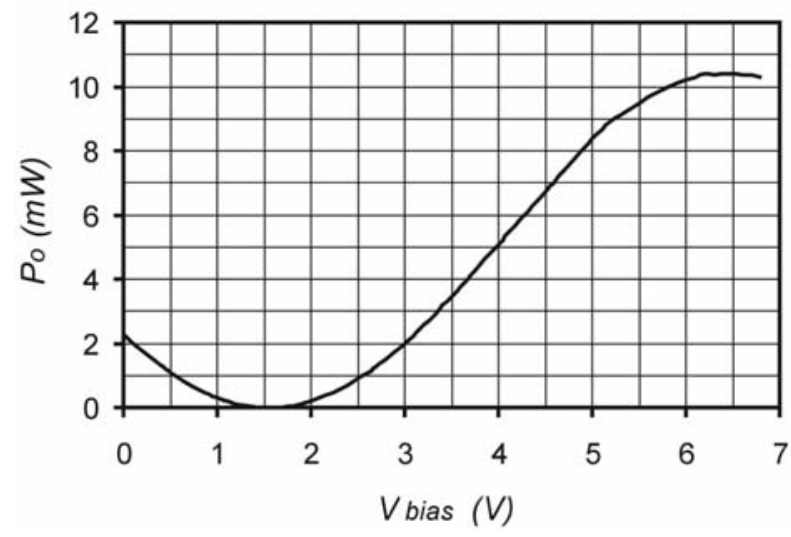

Fig. 8 The MZM transfer characteristic, $P_{o}=f\left(V_{\text {bias }}\right)$, provided

$$
P_{\text {in }}=21.68 \mathrm{~mW}
$$

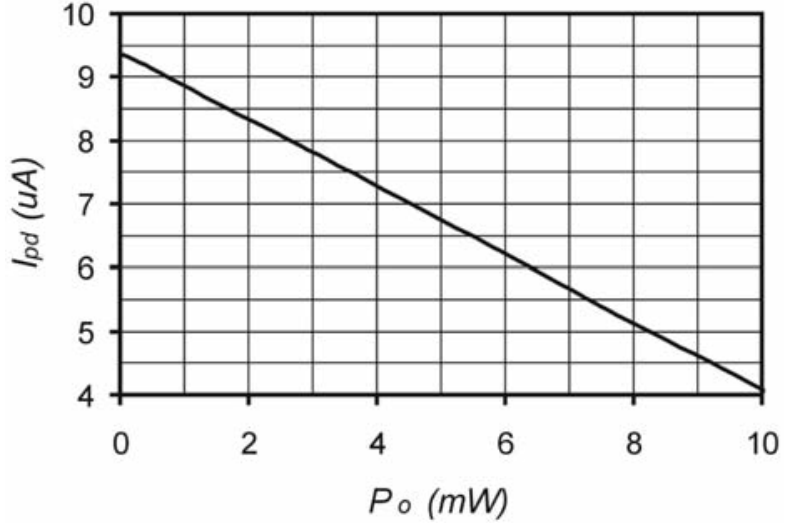

Fig. 9 Dependency of inner PD current on output power, $I_{p d}=f\left(P_{o}\right)$, provided $P_{i n}=21.68 \mathrm{~mW}$

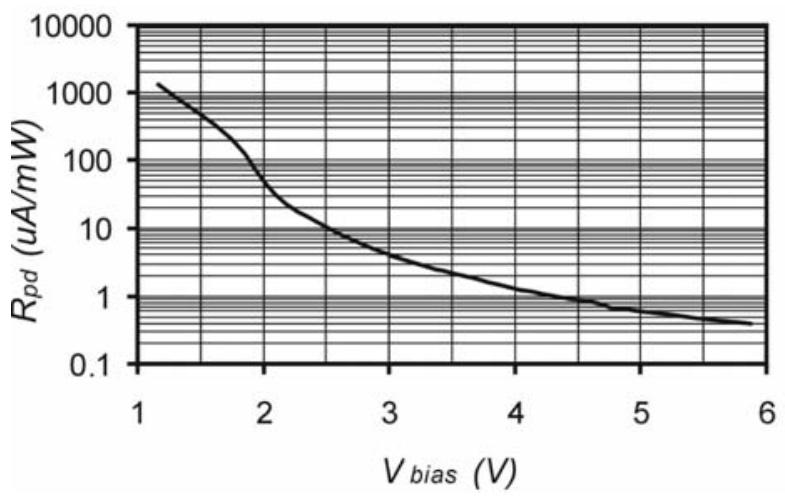

Fig. 10 Dependency of inner photodiode responsivity on set-up operating point, $R_{p d}=f\left(V_{\text {bias }}\right)$

Consequently, charts depicted in Figs. 8, 9 and 10 were plotted using recalculated and measured voltages. The transfer chart view revealed that $\mathrm{DC}$ intrinsic offset is $1.55 \mathrm{~V}$ and output power related

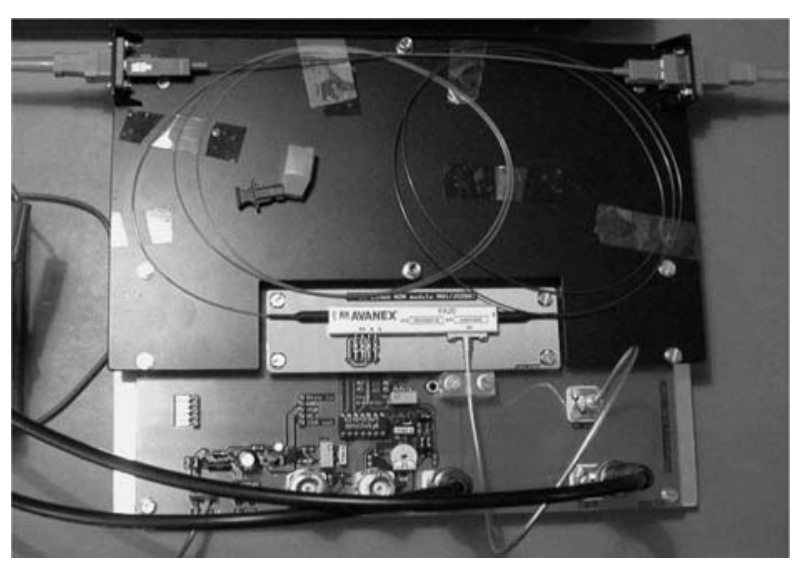

Fig. 11 The base-board with the MZM and auxiliary circuitry 
to the quadrature point adjustment is around $5.2 \mathrm{~mW}$. The dependency in Fig. 9 can be easily described by linear regression (8),

$$
I_{p d}=\eta P_{o}+\delta,
$$

where $\eta=-0.53 \mu \mathrm{A} / \mathrm{mW}$ represents monitor photodiode relative gain and $\delta=9.39 \mu \mathrm{A}$ is photodiode current in case of fully destructive recombination, provided $P_{\text {in }}=21.68 \mathrm{~mW}$. Using data from Figs. 8 and 9, the chart describing dependency of absolute photodiode responsivity on position of operating point (represented by $V_{\text {bias }}$ value) was obtained (see Fig. 10). The chart proves the correctness of measurements. Regard to the data-sheet values there is responsivity $R_{p d}=1.3 \mu \mathrm{A} / \mathrm{mW}$ at quadrature point. This value is in accordance with value found in Fig. 10.

\section{Conclusion}

Important parameters and information necessary to carry-on design of electro-optic remote-antenna system were acquired and verified. There was found the value of intrinsic offset of transfer characteristic. Furthermore, there was found average value of output optical power for quadrature point set-up. Insertion loss value $(\alpha=0.5)$ was verified too.

The measured transfer characteristic, $P_{o}=f\left(V_{\text {bias }}\right)$, helped to specify demands on receiver of the electro-optic system. It is sup- posed that receiver should be designed as a passive one i.e. without any post-amplification. This precaution should help to improve both the flatness of the frequency response and the noise figure of receiving unit as well. That is why the receiver PIN diode should have capability to handle relatively high input optical power (up to 10 $\mathrm{mW}$ ) and simultaneously be able to cover the desired frequency range up to $2.5 \mathrm{GHz}$. This strict requirement is driven by the effort to take a full advantage of high optical gain obtained by MZM unit.

Thanks to linear regression (8) the value of relative sensitivity of inner photodiode to changes of output optical power is well known now. This parameter is very important for design of prospective DC bias feedback loop. Consequently, final result can be declared: If the DC bias feedback system has to be able to keep operating point of the MZM with acceptable error, to say less than $\pm 1^{\circ}$, it translates to necessity to maintain the photodiode current at value of $6.67 \mu \mathrm{A}$ with fluctuations less than $\pm 48 \mathrm{nA}$.

\section{Acknowledgement}

This work was financially supported by Research project MSM 4977751310 of Ministry of Education of the Czech Republic: "Diagnostics of Interactive Processes in Electrical Engineering”.

\section{References}

[1] COX, C. H.: Analog Optical Links-Theory and Practice, Cambridge University Press, 2004.

[2] ACKERMAN, E., ROUSSELl, H., COX, C. H.: Bias Controllers for External Modulators in Fiber-Optic Systems, Photonic Systems, Inc. 2001. http://www.photonicsinc.com

[3] Photonic Systems, Inc.: Modulator Bias Controllers Appl. Note, http://www.photonicsinc.com

[4] Avanex, Inc.: PowerLog ${ }^{T M}$ FA-20, 20 GHz Analog Intensity Modulator with Small Form Factor, Data-Sheet. http://www.avanex.com

[5] SVARNY, J.: Highly Stable $20 \mathrm{~mW}$ Infrared Laser Source. In Proc. Int'1 Conf. Applied Electronics 2009, ISBN 978-80-7043-781-0, Plzen, pp 249-252.

[6] ILX Lightwave, Inc.: Optical Multimeter OMM-6810B User's Guide, InGaAs Power/Wavehead OMH-6727B User's Guide. http://www.ilxlightwave.com. 